\title{
Acta Cirúrgica Brasileira e as Universidades
}

\author{
Saul Goldenberg* \\ * Fundador e Editor Chefe da Acta Cir Bras
}

Ao tomar conhecimento da publicação sobre o Ranking das Universidades na Folha de S.Paulo de 13 de março de 2010, no caderno $\mathrm{C} 4$ Cotidiano $^{1}$ tive a iniciativa de associar a lista da qualidade das instituições com os artigos recebidos pela Acta Cirúrgica Brasileira.

\begin{tabular}{|c|c|c|}
\hline INSTITUIÇÃO & Nota atual & Faixa \\
\hline Universidade Federal de São Paulo & 439 & 5 \\
\hline Universidade Federal do Rio Grande do Sul & 415 & 5 \\
\hline Universidade Federal de Minas Gerais & 413 & 5 \\
\hline Universidade Federal do Triângulo Mineiro & 400 & 5 \\
\hline Universidade Federal do Rio de Janeiro & 390 & 4 \\
\hline Universidade de Brasília & 389 & 4 \\
\hline Universidade Federal de Itajubá-Unifei & 389 & 4 \\
\hline Unesp (5 artigos todos com apoio FAPESP) & 374 & 4 \\
\hline Universidade de Santa Catarina & 372 & 4 \\
\hline Universidade Federal de Pernambuco & 356 & 4 \\
\hline Universidade Estadual de Maringá & 352 & 4 \\
\hline Universidade Federal de Uberlândia & 348 & 4 \\
\hline Universidade Federal de Santa Maria & 347 & 4 \\
\hline Universidade Federal de Goiás & 345 & 4 \\
\hline Universidade Federal do Rio Grande do Norte & 340 & 4 \\
\hline Universidade Federal do Paraná & 340 & 4 \\
\hline Universidade Federal do Rio de Janeiro & 339 & 4 \\
\hline Universidade do Estado do Rio de Janeiro & 339 & 4 \\
\hline Universidade Estadual de Londrina & 329 & 4 \\
\hline Universidade Estadual do Ceará & 329 & 4 \\
\hline Universidade Federal Fluminense & 327 & 4 \\
\hline Universidade Federal da Bahia & 325 & 4 \\
\hline PUC-RS & 321 & 4 \\
\hline Universidade Federal de Mato Grosso do Sul & 314 & 4 \\
\hline Universidade Federal do Espírito Santo & 304 & 4 \\
\hline Universidade Luterana do Brasil & 302 & 4 \\
\hline Universidade de Caxias do Sul & 297 & 4 \\
\hline Universidade de Mato Grosso & 285 & 3 \\
\hline PUC-PR & 278 & 3 \\
\hline Universidade Federal do Amazonas & 276 & 3 \\
\hline PUC-Campinas & 267 & 3 \\
\hline Universidade Federal do Maranhão & 266 & 3 \\
\hline Universidade de Alagoas & 258 & 3 \\
\hline Universidade de Marília & 255 & 3 \\
\hline Universidade Potiguar & 252 & 3 \\
\hline Universidade Federal do Pará & 247 & 3 \\
\hline Universidade São Francisco & 234 & 3 \\
\hline
\end{tabular}

USP e UNICAMP não foram incluídas no ranking. A atuação destas instituições na remessa de artigos para a Acta Cir Bras pode ser verificada em editorial publicado ${ }^{2}$.

\section{Conclusão}

Fatores de impacto:

1. Instituições mais bem avaliadas têm enviado artigos para a Acta Cirúrgica Brasileira;

2. Os artigos enviados são originais e de pesquisa em cirurgia experimental;

3. Comprova-se a produção científica de investigação laboratorial em cirurgia do nosso País;

4. É o melhor retorno ao apoio permanente do CNPq e esporádico da FAPESP.

\section{Referências}

1. www.folha.com.br/1007111

2. Goldenberg A, Fino TPM, Goldenberg S. Acta Cirúrgica Brasileira. Representação interinstitucional e interdisciplinar. Acta Cir Bras. 2009;24(5):334-7. Disponível em www.scielo.br/acb. 